\title{
De los proyectos de trabajo soñados a la realidad del aula en Educación Infantil
}

\author{
Nuria Abal Alonso, Eduardo José Fuentes Abeledo, Pablo César Muñoz Carril \\ Dpto. de Didáctica y Organización Escolar, Universidad de Santiago de Compostela
}

\begin{abstract}
Resumen
Esta aportación surge en el marco de un proyecto de investigación que pretende profundizar, tanto desde un enfoque cuantitativo como cualitativo, en el conocimiento de los procesos y resultados de la formación de maestros y maestras de Educación Infantil en centros universitarios gallegos. Presentamos hallazgos, procedentes de un estudio de caso desarrollado con una aprendiz de maestra. Nos centramos en comentar el contraste que se desvela entre las concepciones que mantiene sobre la relevancia del trabajo por proyectos en Educación Infantil, que identifica como la opción didáctica que mejor se corresponde con su forma de entender el trabajo en las aulas del segundo ciclo de la etapa, y lo que observa que sucede en su primera experiencia de prácticas.

Palabras clave: practicum, educación infantil, enfoque de proyectos, formación inicial de maestros, estudio de caso.
\end{abstract}

\section{Contexto, objetivos y metodología}

Esta aportación es complementaria de otra presentada por los mismos autores a este Congreso. Por limitaciones de espacio, en este texto seremos muy concisos respecto a aspectos comunes de ambas (origen, contexto, objetivos y método de la investigación), enmarcadas en un proyecto de investigación, no subvencionado, centrado en profundizar en el conocimiento de los procesos y resultados de la formación inicial de maestros y maestras de Educación Infantil en el contexto gallego, tanto desde un enfoque cuantitativo como cualitativo.

Esta comunicacón responde a una de las líneas de trabajo en la que pretendemos indagar en torno a la propuesta curricular para formar maestros y maestras de Educación Infantil en la Universidad de Santiago de Compostela (Fuentes Abeledo, 2009) y, más en concreto, identificar, describir y analizar las concepciones, conocimientos dilemas y competencias y su evolución- que mantienen los futuros profesores de Educación Infantil en relación con la tarea docente en esta etapa, así como los elementos que han podido influir en su configuración, centrándonos especialmente en el impacto del programa de formación, sobre todo en relación con el Practicum I desarrollado en el primer cuatrimestre del segundo curso de la carrera.

El estudio de caso (Simons, 2011) ha sido la estrategia metodológica cualitativa por la que hemos optado, situándonos en un marco conceptual de índole hermenéutica, fenomenológica e interpretativa. Los datos recogidos proceden de múltiples fuentes, fundamentalmente de un cuaderno de reflexión y otros documentos de una materia teórico-práctica ubicada en el mismo cuatrimestre que el Practicum I (Documento 1) y otros Documentos (D2 y D3) producidos por Isabel (nombre supuesto de la futura docente que participa en la investigación) a raíz de su experiencia de prácticas (Practicum I), aunque también hemos revisado los registros de entrevistas, grupos de discusión y seminarios de trabajo.

Para el análisis de los datos procedimos a varias lecturas exploratorias y a un proceso de codificación y clasificación de la información analizando minuciosamente su contenido, emergiendo un conjunto de categorías (por ejemplo: finalidades de la Educación Infantil, aprendizaje, niño, maestro, clima de aula, metodología, institución, currículo, modelo didáctico, etc.), y de subcategorías para cada una de las mismas que nos han permitido ordenar las concepciones (diferenciándolas de descripciones neutras, valoraciones positivas o problemas), y dotar de sentido al conjunto para ir desvelando la "perspectiva" de "Isabel". Intentamos, como recomienda Patton (1980) para el análisis cualitativo, "hacer lo más que se pueda para darle sentido -descifrar las cosas-” (p. 345).

Lo que denominamos "perspectiva" constituye, a nuestro entender, un esquema de conceptualización, interpretación e intervención que mantiene Isabel en relación con la práctica educativa en Educación Infantil. En esta comunicación presentamos una primera aproximación a parte de las concepciones de esta futura docente centrándonos en la opción didáctica por la que se inclina antes de su primera experiencia en la escuela -el enfoque de proyectos-, y el impacto que produce en su perspectiva el encuentro con una realidad escolar en el Practicum I. .

\section{Ideal didáctico y realidad en el Practicum I: El caso de Isabel}

\section{Concepciones, imagen ideal y conocimiento para la enseñanza: el enfoque de proyectos como opción curricular fundamentada}

El análisis de los datos desvela que Isabel, antes de incorporarse a su primera experiencia de prácticas en un aula de segundo ciclo de Educación Infantil, ha construido un amplio conjunto de concepciones en torno a la enseñanza que se afianzan tras su primer paso por un colegio como aprendiz de maestra, su conocimiento de experiencias concretas del trabajo con niños pequeños, y su acceso a elementos del pensamiento de su tutora de la escuela. Prestaremos especial atención a la opción curricular que parece orientar su pensamiento, y en torno a la cual articula muy diversos conocimientos que ha ido adquiriendo en el entorno académico universitario, relacionándolos también con algunos otros provenientes de otras fuentes. 
Isabel apuesta por la centralidad del niño en todo planteamiento educativo, a partir de la confianza que tiene en sus potencialidades. Se sitúa en lo que ella misma denomina "perspectiva puerocéntrica" (D1, 43/5). En el primer día de clase de su segundo curso en la Facultad ya afirmaba lo siguiente: "creo firmemente que cada persona tiene un afán de saber, conocer y descubrir" (D1, 30/1). Convicción que se reafirma después de sus primeras prácticas al entender al niño como "una persona con capacidades, potencialidades y derechos” (D3, 30/1).

Esta representación positiva le conduce a defender un proceso de enseñanza basado en el propio infante, un proceso organizado por la maestra pero en el que el alumnado es considerado como "un co-constructor de su conocimiento, de su identidad y de su cultura" (D1,30/1). Isabel defiende que, ante los problemas que puedan surgir se preste suma atención a la realidad de cada niño y del grupo concreto de alumnos; que se profundice "en la individualidad del sujeto supliendo carencias y potenciando la personalidad y creatividad" (D1, 14/9). Ella piensa que algunos maestros para solucionar dichos problemas "ignoran al elemento primordial”, que es “el niño” (D1, 14/9).

Tras la experiencia en las aulas manifiesta con firmeza, al igual que declaraba previamente a la misma, la necesidad de que la escuela preste atención a las necesidades e intereses de los niños ("el interés del alumno es el principal impulsor de su desarrollo” -D1, 45/5-, llega a decir), se adapte "a la concreción del pensamiento infantil” (D1,30/4) y a "la diversidad de ritmos de aprendizaje empleando el juego y las experiencias cotidianas como medios de su efectivización” (D1, 30/4).

El maestro, piensa Isabel, ha de tener en cuenta las características específicas de cada alumno, reconociendo, en alusión a la teoría de Gardner, la “diversidad de inteligencias" (D1, 12/3), y "tiene la obligación de fomentar el ser resolutivo y competente con el fin de que adquieran confianza y experiencia (D1, 14/7), apostando por la motivación intrínseca"'(D1, 12/7 y 28/8) y un clima de aula distendido, en donde florezcan la confianza, la colaboración y el bienestar emocional facilitando el compromiso e implicación con las actividades que faciliten el aprendizaje "significativo" y "profundo" y el crecimiento personal en todas las dimensiones.

Ella cree que es posible generar en la escuela de Educación Infantil actividades que permitan que se aprenda con interés y pleno sentido para cada niño expandiendo las posibilidades de crecimiento de los pequeños.

De ahí su entusiasmo ante propuestas curriculares que despierten el gusto por aprender, que impliquen a los escolares trabajando con contenidos muy diversos, partiendo de problemas cotidianos o que puedan interesar a los pequeños y también al profesorado, responsable este último de "escuchar" a los pequeños y generar experiencias y procesos que atraigan motivando hacia el aprendizaje profundo y significativo.

Isabel encuentra en el enfoque de proyectos una respuesta adecuada para hacer posible en la práctica de aula ideas como las que hemos sintetizado en párrafos anteriores, ligando "el concepto de proyecto" al "interés del alumno", que considera "el principal impulsor de su desarrollo por lo que para estimular el campo cognoscitivo, es preciso asentar el proceso de enseñanza-aprendizaje sobre la curiosidad infantil, la afectividad y la utilización de diferentes recursos disciplinares" (D1, 45/5).

Esta aprendiz de maestra, tras extenderse en comentar lo que entiende por enfoque de proyectos, resume su concepción con estas palabras: "un conjunto de experiencias de aprendizaje atractivas que involucran al alumnado en vivencias propias del mundo real aplicando las habilidades y conocimientos adquiridos. Es una estrategia que reconoce que el aprendizaje significativo lleva al alumnado a un proceso inherente de aprendizaje, a una capacidad de hacer trabajo relevante. Esto implica un manejo de informaciones interdisciplinares, necesarias para poder emplear los recursos de los que disponen en el aula y en el medio y desarrollar habilidades y destrezas. En consecuencia, se relaciona con la resolución de problemas, resolver preguntas buscando respuestas y otras tareas significativas, beneficiando el trabajo autónomo para la construcción del propio aprendizaje, culminando en resultados reales generados por ellos mismos" (D1, 46/3).

Este planteamiento conlleva, según expone Isabel, "superar los límites curriculares” (D1,46/1), apostando por un "currículo integrado que se sustenta en el concepto de globalización y en la interdisciplinariedad del conocimiento, de modo que la integración del saber puede ser facilitada con una presentación acorde a la intencionalidad" (D1, 48/2). Defiende que "en los métodos globalizados el organizador principal es el alumnado y sus necesidades educativas” (...) este proceso globalizador tiende a establecer relaciones entre las disciplinas favoreciendo la capacidad de relacionar. Con este proceso se atiende a la naturaleza relacional del aprendizaje, a la naturaleza global de las disciplinas así como a la resolución de problemas curriculares de diferente grado de dificultad” (D1, 48/2). De esta forma, $\mathrm{y}$ aunque se empleen "instrumentos conceptuales y técnicos disciplinares", se posibilita la "proximidad a la realidad del alumno, a sus intereses y curiosidad infantiles" (D1, 48/3).

Desde su perspectiva y en relación con la selección de los contenidos, se han de incorporar tanto aquellos de orden "conceptual", como "procedimental” y "actitudinal” de forma equilibrada (D1, 36 y 37). Advierte antes de las prácticas que, sin embargo, "contamos con un sistema educativo que prima lo académico sobre lo actitudinal de forma que los infantes cuentan con un saber estar y hacer en la escuela que depende del clima de aula establecido y del compromiso formativo del profesor. Es preciso realizar un trabajo diario sobre estos aspectos, aunque sea de forma inconsciente, ya que tácitamente son transmitidos y por lo tanto reproducidos por los discentes (D1, 9/2).

En todo caso, advierte que hay que estar alerta en relación con la tendencia al "academicismo" (D1, 39/2) en la selección de los contenidos. Isabel descubre 
grandes posibilidades para trabajar en la Educación Infantil en la orientación curricular que apuesta por ofrecer "un currículo mucho más variado y vinculado a la experiencia de los sujetos", incorporando "todo aquello que constituya un ámbito del conocimiento o experiencia susceptible de ser enriquecido desde la escuela. Consecuentemente, los criterios de necesidad y actualidad se revalorizan debido a la incorporación de ámbitos del conocimiento pertenecientes al 'aquí y ahora' así como la vida diaria del alumno” (D1, 39/3).

Así, pues, el motivo principal por el que Isabel opta por el enfoque de proyectos como una opción curricular, se concentra sobre todo en torno a la respuesta que ofrece a las necesidades e intereses del alumnado, respetando su naturaleza, situando al infante como el eje en torno al que debe pivotar la enseñanza, en una perspectiva acorde con lo que defendía la "Escuela Nueva”. Esta orientación facilita además la atención a la diversidad, la expansión "de las facultades creativas y de originalidad recordando la plasticidad y el dinamismo del medio en el que interviene activamente" (D1, 27/4), y la emergencia de un clima de aula en el que las relaciones de los niños con el profesor y de los niños entre sí, sean de calidez, confianza, cordialidad y afecto potenciando además actitudes democráticas, ajenas a una relación de poder-sumisión, y en donde prime la colaboración, la solidaridad y la cooperación, la responsabilidad y el compromiso.

Los proyectos, defiende Isabel antes de su experiencia en las prácticas: “alimentan el diálogo y la aceptación de la diversidad y de la individualidad de cada sujeto, garantizando la implementación del cambio y de las diferencias. Este sistema de gerencia participativa posibilita y agiliza la asunción progresiva de obligaciones, democratizando el aula e incrementando el sentido de compromiso y responsabilidad con el maestro, con los compañeros, con la escuela, con la familia y consigo mismo. De este modo, se erige una perspectiva compartida en la que el docente desempeña el papel de guía” (D1, 27/2).

Por otra parte, el enfoque de proyectos, tal y como lo entiende Isabel, le permite también mantener la coherencia con otra concepción muy asentada en su pensamiento y referida a la necesidad de abordar muy diversos contenidos, de forma integrada y respetando el principio de "globalización”. Como afirmaba, en el primer mes de clases de segundo curso: "Creo firmemente en la importancia de la música, plástica, dramatización y psicomotricidad (D1, 12/3). El enfoque de proyectos, según ella, facilita el trabajo con contenidos como los citados, a veces, afirma, injustamente olvidados en la vida de las aulas de Educación Infantil. Con carácter general, además, para Isabel los proyectos permiten el "estudio profundo de temas del mundo real” (D1, 27/7).

Por otra parte, refiriéndose al ámbito del profesorado, Isabel señala otro motivo que justifica su inclinación al enfoque de proyectos y que se deriva de concebirlo como una incitación "al compromiso real con los procesos de cambio, al trabajo en equipo, al desarrollo de visiones compartidas $y$ al intercambio de experiencias de aprendizaje y de desarrollo personal”
(D1, 27/7). Los maestros y maestras, desde su punto de vista, han de ser reflexivos, investigadores de la práctica, huyendo del anquilosamiento y la rutina.

Apostar por el enfoque de proyectos hace posible esta idea de profesor puesto que lo implica como investigador ante el objetivo de que también sus alumnos investiguen, generando para ellos espacios de "investigación, experimentación, manipulación y observación” (D1,16/2), actuando el docente como guía del proceso.

Como escribía Isabel al comienzo de su segundo curso de carrera, el maestro ha de ser un profesional capaz de argumentar y de no sentirse colonizado por materiales editados (D1, 2, 3 y 4). Un profesional, como declara semanas después, preparado para formular "actividades de investigación, de construcción y de dramatización que consigan generar una experiencia lúdica y enriquecedora a través de un tópico estimulado y surgido de las motivaciones del alumnado” (D1, 28/5), y capaz de "particularizar el tópico y los contenidos a través de estímulos motivadores utilizando las ideas, dudas e intereses de los niños con el fin de desarrollar un aprendizaje significativo" (D1, 28/5).

Además, desde su perspectiva, el enfoque de proyectos facilita la aproximación a las familias y potencia un mayor compromiso de las mismas con el proyecto de la escuela. Isabel, tras la experiencia de prácticas, se reafirma en la necesidad de que el profesorado desarrolle "un trabajo comunicativo y participativo con la comunidad escolar” (D1, 29/7), de forma que sus planteamientos sean conocidos y debatidos con los padres. Así, por ejemplo, entiende que la orientación hacia el enfoque de proyectos, dadas sus virtudes, puede constituir una opción compartida por los progenitores, potenciándose la implicación y colaboración en diferentes actividades ligadas al mismo.

\section{Vivencia cotidiana y estructuras de aula: del ideal a la realidad en la escuela de prácticas}

En la vida del aula, la "práctica docente" puede entenderse como el conjunto de actividades, tareas y rutinas que van configurando, en su dinámica cotidiana, un determinado patrón de acción habitual. Dicho patrón suponemos que responde a una determinada intencionalidad del docente que afronta el trabajo con un grupo específico de alumnos. Uno de los aspectos que nos permite entrar en el análisis de lo que sucede en un aula concreta, y del patrón de acción del docente, es la estructura temporal y espacial que se adopta.

Isabel, como hemos comentado, ha ido construyendo una determinada visión de la práctica docente ideal en Educación Infantil, considerando el niño como el eje educativo, y entendiendo el enfoque de proyectos como la opción curricular más adecuada. Pertrechada con un conjunto de concepciones, algunas de las cuales hemos presentado en párrafos anteriores, se sumerge en la cotidianeidad de una escuela, en un aula concreta de una localidad gallega.

La futura maestra pretende captar, analizar e interpretar el patrón docente que caracteriza la vida del aula de la que participa durante un mes de estancia en el 
colegio y llegar a entender, en relación con el mismo, la intencionalidad de su tutora sirviéndose también de los datos procedentes de conversaciones con la docente y del análisis de documentos diversos ligados a la vida del aula (como "programaciones” y otros documentos de planificación que usa la profesora). Este planteamiento de Isabel se manifiesta explícitamente cuando declara, por ejemplo, que "la organización del tiempo, al igual que la del espacio, está correlacionada con la intencionalidad educativa de la maestra” (D3, 22/1), o cuando dice que "la organización espacial es el prefacio del análisis de la acción del profesorado, al implicar una relación directa con la intencionalidad docente” (D1, 12/7).

El análisis de la secuencia de actividades de la vida del aula que Isabel lleva a cabo tras la recogida minuciosa de datos durante su estancia de prácticasdesde la entrada y salida en filas hasta los diferentes momentos que suelen sucederse entre ambas-, le lleva a declarar que se sigue una pauta repetitiva, día tras día. Isabel se detiene en descifrar lo que en conjunto denomina "estructura rutinaria" informando que hay también momentos “de especialidad” (horas específicas para religión, música, psicomotricidad e inglés con intervención de otros profesores diferentes a su tutora, y que habitualmente se desarrollan en espacios distintos al aula del grupo). Su análisis le conduce a comentar que dicha estructura, y una buena parte de las actividades concretas, "se encuentra lejos de incitar el interés infantil” (D3, 24/3), considerándola alejada de propuestas que respondan a lo que ella defendía antes de las prácticas, y en las que insiste de nuevo durante su estancia en el centro ("la lógica de lo contextual y situacional”-D3, 24/3-).

La estructura que se revela le parece alejada de las concepciones que defiende, afirmando, por ejemplo, que "la organización del tiempo lineal implica unas transiciones muy definidas de modo que el ímpetu y la voluntad de aprendizaje se reducen debido a la falta de libertad expresiva intensificada por la rigidez”. Isabel comenta que esto provoca en muchas ocasiones un “ambiente disruptivo entre actividades", lo que señala como "un claro indicador de la falta de calidad de las experiencias ofrecidas en las rutinas y en el aula” (D3, 24/4).

Desde la interpretación de Isabel la organización pivota en torno a la realización de las "fichas", con tareas “de lápiz y papel”, revelándose, en su opinión, una clara "jerarquización temporal de las actividades": por la mañana lo importante (las fichas), por la tarde, “actividades complementarias”. Isabel destaca, acorde con lo que hemos señalado respecto a sus concepciones y a la apuesta por el enfoque de proyectos, que "no existe una visión integrada y global de las actividades" (D3, 24/3).

De la clase que observa subraya el hecho de que "las rutinas priorizan los tiempos dedicados al lenguaje y a la matemática. De índole rígida y lineal, la propuesta rutinaria (...) es una prolongación de los déficits espaciales ya que a su estrecha conexión visualizaba los aspectos más favorecidos. Así, el espacio dedicado al recreo, y por consiguiente al patio, era un mero espacio de juego infantil, en vez de ser un área de observación e interacción con los sujetos. El excesivo tiempo destinado a ambas áreas -lenguaje y matemáticas- se contrarrestaba con una desatención a otros ámbitos de igual o superior importancia de modo que esas aprendizajes eran eximidas” (D3, 25/1).

Por otra parte, la realización de las fichas se desarrolla, desde la visión de Isabel, con una fuerte “presión temporal”, dificultándose la “adaptación gradual del niño a las diferentes situaciones”, a su propio ritmo, provocando que los niños más lentos se sintiesen "nerviosos e intranquilos", sobre todo aquellos "con mayores dificultades" (D3, 24/2).

Espacio.

La interpretación que Isabel hace de la organización espacial de la clase en buena parte responde también a su concepción del niño como eje de la acción educativa: "las áreas no se organizan teniendo en cuenta aspectos prácticos ni los cambios de interés de los niños, así como la variedad de experiencias lúdicas y los desplazamientos infantiles” (D3, 17/1); con escasa atención a "la satisfacción de las necesidades educativas del niño (...), a las motivaciones e intereses infantiles" (D3, 18/2). Necesidades, como por ejemplo, “de placer, acción, movimiento, autonomía e iniciativa personal, experimentación, contacto" (D3, 29/4). Necesidades que, piensa esta futura docente, no se atienden en la medida de lo deseable, favoreciéndose, sin embargo, "el papel dependiente del alumno" (D3, 29/4).

Desde su punto de vista, todo el entramado espacial del aula que observa depende del enfoque del "trabajo con fichas" al que se adhiere la tutora: "tiene unos espacios a los que ofrece mayor atención debido a la complementariedad con las fichas" (D3, 17/1) y lo mismo sucede con el uso de los materiales que en ellos se encuentran y que desde la visión de Isabel, se limitan a "una labor subsidiaria de las fichas" (D3, 17/2).

Desde estos planteamientos, si bien Isabel reconoce el valor de organizar "rincones" (ella los describe con detalle, al igual que el material y dinámica y actividades concretas que se desarrollan habitualmente en los “rincones” de: “juego simbólico”, “lenguaje artístico”, “nuevas tecnologías”, y "lógico-matemático”, además el espacio de "biblioteca"), y manifiesta que "esta distribución es propia de un enfoque de proyectos” (D3, 15/7), en el caso concreto de su aula de prácticas lo valora como un mero "conato de organización por rincones” (D3, 13/3), al entender que la "metodología educativa" de la tutora (centrada en la realización de numerosos "fichas" y de trabajo con lápiz y papel, y todo ello muy centrado en contenidos ligados al aprendizaje de la lecto-escritura y nociones matemáticas), no es coherente con el uso que de esos espacios se hace en un enfoque de proyectos, manifestándose crítica con diversos aspectos del desarrollo de la vida del aula que observa, reconociendo también en positivo aquellos aspectos que se corresponden con las concepciones y principios que mantiene. Así, por ejemplo, afirma que los materiales están dispuestos según principios como "accesibilidad y visibilidad”, o que están correctamente clasificados y etiquetados y que en su uso se manifiesta el respeto a 
unas normas de conservación y utilización -surgidas de la práctica, consensuadas y repetidas- (D3, 17/3).

Desde la perspectiva de Isabel, desde su marco interpretativo, diversas declaraciones de la tutora en relación con la intencionalidad de sus acciones (como organizar las mesas para el "trabajo de grupo" -D3, 12/8- o permitir momentos de “juego" -D3, 16/2-) resultan incoherentes con lo que realmente sucede en el aula. Para esta futura profesora, si bien los niños "están sentados en grupo (...) no desarrollan un trabajo en equipo destacado por la cooperación y la colaboración entre iguales. Así, las mesas y las sillas eran habitualmente intuidos como elementos de sujeción del ente infantil en el que realizar el trabajo individualizado" (D3, 12/8), comentando en variadas ocasiones a lo largo de su estancia de prácticas que, desde su perspectiva, el ambiente reinante es de “competitividad”. Isabel destaca también la distancia entre la intención y la realidad en relación con el tratamiento del juego, reflejándose en su opinión una clara "dicotomía entre juego y trabajo” (D3, 13/3), pues si bien la tutora le declara que "intento organizar mis clases en torno al juego" (D3, 16/2), desde la perspectiva de Isabel, su tutora "propicia el juego como una realidad ajena al aula de modo que no lo formula como una vía de capital transcendencia en los aprendizajes discentes. Creo que la combinación de rincones con fichas es algo vacío y carente de sentido ya que en su antítesis formulan principios contrarios. Así la organización espacial por rincones implica un respeto cara a los ritmos de aprendizaje del alumno, a la diversidad, a la promoción del trabajo en equipo, a la comunicación y participación conjunta, a la promoción del sentimiento de competencia y de la capacidad de autorregulación del niño, a la individualización de la enseñanza" (D3, 16/2). En definitiva, pues, no se reflejan en la vida del aula aspectos que ella considera inherentes a la organización por rincones que, a su vez, liga al enfoque de proyectos.

Por otra parte, Isabel, defensora de un enfoque de currículo integrado en el marco del desarrollo de proyectos, descubre en su primera experiencia en la escuela como aprendiz de maestra que el mismo grupo de alumnos tiene varios profesoras más (de religión, psicomotricidad, música e inglés). Cada una de estas docentes desarrolla sus clases en unos horarios fijos a lo largo de la semana, y en unos espacios específicos a los que se desplazan los niños, espacios, en ocasiones diferentes al de las clases con su tutora -excepto en religión-. Estas actividades, junto con la realización de tareas en cuadernos centrados en los aprendizajes ligados a la lecto-escritura y a las matemáticas, componen el currículo fundamental que se desarrolla en el aula a la que acude Isabel y que ella misma encuadra en una orientación "logocéntrica”. Un currículo que, en cuanto a su estructura, encuadra en el bloque de aquellos que se organizan "por yuxtaposición, segmentación o disciplinariedad” (D3, 30/1). Para ella, "didáctica y curricularmente los contenidos eran descontextualizados e inconexos, priorizando objetivos instructivos relacionados con la lectura, escritura y el aprendizaje de la numeración” (D3, 30/3)
En la clase a la que acude Isabel la realización de múltiples "fichas" por parte de los niños se convierte en la actividad nuclear y a la que se dedica más tiempo. La la misma tutora le manifiesta que, en ocasiones, "están bastante apurados” y con presión para terminar las que están establecidas para un determinado periodo, destacándose el objetivo de poder "llevarlas para casa" (D3, 22/1) y que las vean los padres de los niños. Desde la perspectiva de Isabel los "rincones", y las actividades que allí se realizan, se convierten instrumentos para el "refuerzo extrínseco" cuando el alumnado termina la ficha correspondiente (D3, 18/ 2 y 3), pero sin estar integradas en un proyecto con otras intencionalidades explícitas.

Tras su estancia de prácticas, Isabel se reafirma con contundencia en su defensa de la opción por un enfoque de proyectos que entiende "antagónica" de lo que ha visto en la vida del aula. Una opción que se fundamente en "un puerocentrismo que prevalezca y garantice la satisfacción de las necesidades infantiles sobre la operativización de objetivos” (D3, 40/2). Otras muchas declaraciones no dejan lugar a dudas sobre las concepciones que mantiene, aportando principios y ejemplos de propuestas didácticas. Por ejemplo:

"Considero que la concreción del derecho a la educación debe llevarse a cabo en una '”escuela por y para la vida”, institución en la que considero que la aplicación del enfoque de proyectos desempeñaría un eficaz $y$ notorio papel en el trato de la multidimensionalidad y simultaneidad de aprendizajes así como en la inmediatez e imprevisibilidad" (D3, 39/1).

"Partiendo de los lemas constructivistas, considero que en el aula y en el centro escolar se deben incrementar el número de experiencias relacionadas con la vida diaria, como por ejemplo poner la mesa o hacer pan, ya que además de promover procesos de autonomía y resolución de problemas prácticos, sirven para desarrollar de modo continuado el concepto vigotskyano de ZDP (Zona de Desarrollo Próximo), incrementando por ende la plasticidad cerebral. Este proceso, debería llevarse a cabo mediante un proceso planificativo de construcción compartida con el alumnado, que aportando sentido, genere un ambiente motivador que favorezca la ampliación del conocimiento mediante actividades de investigación, construcción y dramatización que partan de experiencias reales o auténticas. Para su aplicación, no es solo precisa la lógica y la congruencia científica en cuanto al currículo sino también un pragmatismo didáctico, organizativo y psicosocial que implique una propuesta curricular innovadora consolidada” (D3, 40/2).

\section{Conclusiones}

\section{La distancia entre pensamiento y acción: un puente para la reflexión}

La estructura habitual de las clases en el aula que observa Isabel, el patrón de acción que se desarrolla responde a una tradición bastante extendida y que se ha venido asentando en la Educación Infantil en nuestro contexto. Lo que ella denomina "el trabajo por fichas", 
la organización temporal y espacial en la que nos hemos detenido en esta aportación, y elementos del currículo desarrollado como los que hemos destacado responden, a grandes rasgos, a un modelo que es preciso comprender considerando también variadas condiciones que fomentan su asentamiento. Isabel destaca la distancia entre algunas concepciones declaradas de la profesora tutora y la realidad en diversos momentos de su estancia en prácticas. Así por ejemplo, escribe: "la homogeneización de la enseñanza llevada a cabo por el sistema de fichas pone en entredicho la intencionalidad educativa de la tutora” (D3, 16/2).

La futura maestra reclama la necesidad de "teorizar": "(la tutora) no considera precisa la consulta de un marco teórico que sustente y argumente su toma de decisiones" (D3, 33/4), de "reflexionar" para analizar los resultados reales con la propuesta que se implementa en la práctica comprendiendo "la diversidad de resultados" (D3, 18/5) y desvelando cómo determinadas concepciones y principios de actuación declarados no se aplican en el aula, pero sirven como discurso políticamente correcto. Para Isabel, como comentamos en otra comunicación que presentamos en este mismo Congreso, desprenderse de la dependencia de currículos editados y volcarse en una planificación que atienda a la "lógica de lo contextual, de lo situacional" (D1, 12/6), como ya defendía antes de su inmersión en las prácticas, es imprescindible para superar incoherencias entre pensamiento y acción.

En el libro de la experimentada y reconocida maestra Carmen Díez Navarro (2008) se presentan interesantes reflexiones ante lo que se denomina en la obra "la eterna contradicción entre el discurso del hacer y el discurso del decir" que "en educación cobra unas dimensiones singulares y muy determinantes” (p. 195) y que, se reconoce, están extendidas entre los docentes de Educación Infantil. Desde la visión de Isabel son varias las "contradicciones" detectadas en su experiencia de prácticas. Su discurso se articula en torno a la perspectiva que ha ido construyendo antes de la experiencia de las prácticas con una fuerte influencia de lo interiorizado en la Facultad de Educación y ligada a teorías, investigaciones y experiencias basadas en el enfoque de proyectos, en el juego, el desarrollo de la autonomía en los niños, el movimiento, etc. Esa perspectiva le sirve de lente interpretativa entrando de lleno en la disección de las contradicciones detectadas. Como se comenta en el libro anteriormente citado, al profesorado en ejercicio esas "contradicciones" suelen producirle una "una gran culpa, que escondemos con vergüenza o una racionalización justificadora, de pretensión pedagógica, que intenta disculparnos y que siempre nos delata” (p. 195).

Isabel, en sus primeras experiencias de prácticas, todavía no ha actuado con autonomía para tomar decisiones docentes, salvo en momentos puntuales y, sobre todo, colaborando con la profesora en las tareas que ella le ha indicado. Por tanto, no ha entrado de lleno en la práctica como protagonista docente principal. Ha sido, fundamentalmente, observadora e intérprete de una realidad concreta, y su experiencia responde a los objetivos básicos del programa del "Practicum I" de su universidad. Sus datos, análisis e interpretaciones pueden ser útiles para alimentar la reflexión sobre las contradicciones citadas, pero también sobre las contradicciones de un sistema formativo universitario y de los propios formadores que también, en no pocas ocasiones, se ven inmersos en incoherencias como las del resto del profesorado de otras etapas. Ayudar a comprender el porqué de las mismas, eso sí, yendo más allá de los límites del aula, resulta imprescindible para propiciar le mejora de todos los implicados facilitando un mayor nivel de coherencia entre pensamiento, acción y sentimiento.

\section{Virtudes y peligros de los modelos idealizados en la universidad}

Hemos constatado el "choque" con la realidad del modelo ideal, de la identidad soñada de Isabel al realizar su primera experiencia en la escuela. La imagen que ha ido forjando de "una buena clase de Educación Infantil" no se corresponde, desde su perspectiva, con el desarrollo de la vida del aula que observa. Es interesante constatar el esfuerzo que esta futura docente hace por penetrar en el significado, en las intenciones y concepciones declaradas de su tutora para indagar qué sucede, para comprender la perspectiva de quien actúa cotidianamente, en un determinado entorno y condiciones.

En este sentido, queremos subrayar el hecho de que potenciar la reflexión en profesores y futuros profesores, puede incitar a cuestionar las concepciones y prácticas de uno mismo y otras a las que se tiene acceso. En el caso de Isabel se ha reafirmado contundentemente en sus concepciones iniciales previas a las prácticas y en la defensa del "enfoque de proyectos".

Manteniendo todas las cautelas y condiciones de confidencialidad, defendemos que dicho proceso de reflexión favorece un análisis de la realidad sobre el qué, para qué, por qué, cómo y cuándo enseñar y evaluar en la escuela infantil, y puede propiciar el impulso hacia la introducción de mejoras y el desarrollo progresivo de competencias docentes acordes con el modelo de docencia que se estime más apropiado si las distintas experiencias de prácticas lo favorecen. Recordemos que las vivencias de Isabel en la escuela se corresponden con el Practicum I y aún le queda un largo camino por recorrer en los Practicum II y III y en las materias teórico-prácticas del segundo cuatrimestre del segundo curso y de tercero y cuarto.

Pero también es preciso subrayar los peligros de que una visión generada en la formación inicial académica que no tenga en cuenta las condiciones en que se despliega la práctica cotidiana y que la han constituido históricamente, pueda conducir a los futuros docentes a frustraciones mayores en el momento en que tengan que tomar decisiones con cierta autonomía. Cabe reseñar igualmente el riesgo de que se pueda propiciar la asunción de discursos de desautorización muy alejados de las posibilidades reales de cambio y mejora, y sin tener en cuenta que el profesorado construye sus respuestas a los complejos problemas de la práctica acudiendo a múltiples referentes y fuentes, sin la 
"pureza” en que habitualmente se mueven los modelos teóricos presentados en la universidad.

Estos riesgos y peligros creemos que no deben inducir a eludir la definición en las instituciones universitarias de formación inicial de un perfil de docente potenciando el ánimo para comprometerse con una perspectiva sobre la Educación Infantil que responda a un enfoque bien fundamentado, a un enfoque de calidad. En todo caso, cualquier propuesta explícita al respecto siempre ha de ser motivo permanente de análisis y debate. La existencia de la misma permite orientar iniciativas formativas para, en un proceso sin pausa de investigación-acción, facilitar una mejor atención a la infancia. La colaboración entre la universidad y la escuela en dicho proceso puede favorecerla.

\section{Referencias}

Díez Navarro, C. (2008). Mi escuela sabe a naranja. Estar y ser en la escuela infantil. Barcelona: Graó. Fuentes Abeledo, E. (2009). Formación de maestros y Practicum en el contexto de cambio curricular desde la perspectiva de la convergencia europea. En Raposo Rivas, M.; Martínez Figueira, M.E.; Lodeiro Enjo, L.; Fernández de la Iglesia, J.C. y Pérez Abellás, A. (Coords), El practicum más allá del empleo: Formación vs. Training (pp.103-124). Santiago de Compostela: Andavira.

Patton, M. (1980). Qualitative Evaluation Methods. Londres: Sage Publications.

Simons, H. (2011). El estudio de caso: Teoría y práctica. Madrid: Morata. 